\title{
Schedule-oriented navigation system for travelers using an urban public transportation network
}

\author{
N. Ozaki, M. Nozue \& R. Tsuchiya \\ Railway Technical Research Institute, Japan
}

\begin{abstract}
In this paper, we describe the design concept of the traveler support system, which can automatically rearrange the itinerary in correspondence with the change of an activity schedule. We also make an account of the result of the experimental operation of the prototype system that we have developed.

Keywords: navigation system, schedule-oriented, revising itinerary.
\end{abstract}

\section{Introduction}

Travelers typically collate information related to their journeys from different information sources and integrate them to make their travel decisions. Recently, web-based information services are commercially available for travelers to search for the route and time to get to their destinations by using an urban public transportation network, including trains and buses.

In order to obtain necessary information, we have to input a departure place, a destination, departure/arrival time into the system, which provides us with some travel plan options. However, it is often the case that we have to rearrange our travel itineraries due to the change of activity schedules (e.g. change of meeting time) or other reasons (e.g. failure to catch the train). In these cases in order to reschedule our travel itinerary, we have to input necessary information into the system again. If we are on the way, we need to confirm where we are. This procedure is troublesome.

If there is a system to rearrange itinerary promptly according to the revised activity schedule, taking into consideration the location of travelers, the convenience of the traveler will be greatly improved. 
Since this type of system is not available now, we have carried out research and development related to a traveler support system, which can flexibly support travelers even in the case of rescheduling.

In this paper, we describe the design concept of the traveler support system, which can automatically rearrange the itinerary in correspondence with the change of an activity schedule. The system automatically traces travelers' location by GPS, and accesses various information sources by a cellular phone network. We also make an account of the result of the experimental operation of the prototype system that we have developed.

\section{Design concept of the system}

In this section, we present a design concept of the traveler navigation system and goals of the system development.

The main function of the system is guidance of the itinerary taking into consideration the schedule and location of a traveler. In addition, the system has four unique functions, which will be discussed in the later sections.

\subsection{Rearranging an itinerary automatically}

The system rearranges itinerary promptly according to the revised activity schedule (e.g. change of a meeting time or cancellation of a meeting), taking into consideration the location of a traveler. Even when a traveler arrives behind schedule in a station and can by no means catch his/her train, the system will look for other travel plan options and proposes them to him/her.

\subsection{Creating a travel itinerary based on activity schedule}

The system automatically creates a travel itinerary for a traveler for the whole day, taking into consideration his/her activity schedule (i.e. when and where each activity takes place) that has already been registered to the system in advance.

Each item in a schedule can be classified into three categories based on the degree of time constraints; (1) fixed item, (e.g. business meeting), (2) floating item (e.g. office machinery maintenance services), (3) loosely-constrained items (e.g. visiting a bank for its counter service).

The system considers these conditions and makes a travel itinerary according to travelers' input related to their activity schedules (e.g. visiting times and places).

\subsection{Telling how much time the user spare for a side trip}

The system can notify travelers how much spare time they will have during their journey. The spare time for a given travel itinerary is the difference between the time when a certain scheduled item (e.g. meeting) will take place and the estimated arrival time when the traveler goes without side trips. The travelers, with the knowledge of the spare time available for them, can always take proper actions during their journey, resulting in the effective use of their time. For 
example, a traveler can tell whether they need to be in haste or not to catch his/her train or whether he/she has some extra time at the transfer station to do some small shopping, etc.

\subsection{Using real-time information}

The system incorporates real-time information obtained from the bus location system as well as the information on the disturbance of train operations in order to provide travellers with up-to-date travel guidance. The bus location system tells us where a bus is running and when a bus comes to a bus stop. If the bus location system is available for guidance, a transfer between the train and bus can be smoother, and each traveler will be provided with the optimized route involving multiple modes to his/her destination.

If the information on train operation disturbances is available, the system will emit a warning and can propose an alternative itinerary, which may contain a detour route and notify them with the estimated arrival time at the destination.

\subsection{Summary of the functions of the system}

Based on the design concepts of the system described above, we have discussed the possible interactions between the system and a user (traveler). Table 1 summarizes the actions, which the system can take, in response to the behaviour of a user under a certain situation. Fig. 1 describes the component of the system.

If we can fully implement these functions into the system, a traveler has only to input his/her activity schedule in order to enjoy the services provided by the schedule-oriented navigation system.

\section{Implementation issues}

It is difficult to implement all functions described in Section 2. Then, we first implement a function that rearranges the itinerary promptly according to the revised activity schedule, taking into consideration the location of a traveler, described in 2.1. There are two main technical issues, which we have to cope with, regarding the implementation of the system.

First, the system cannot always detect the actual location of a traveler due to the geographically restricted availability of location services (e.g. GPS, cellular phone-based location, wireless LAN). It is often the case that, the system cannot detect the traveler's location due to the lack of available location technologies and therefore cannot calculate the itinerary optimized for him/her.

We carried out a preliminary test in which we investigated the availability and accuracy of three typical location detection systems; GPS, cellular phone based location information and wireless LAN, along some lines of railways and buses. As a matter of course, the system cannot receive the signal of GPS satellite under ground and it was accurate when we were on the ground and the system could receive the signal very well. However, it was not accurate when we were between tall buildings on the ground because an insufficient number of GPS satellites can be detected. Although cellular phone based location information 
demonstrated a sufficient level of availability, it only showed an accuracy of approximately 100 meters, which is often insufficient for traveler guidance. Wireless LAN based location services currently suffer from restricted availability. They are available only in major railway stations and in some densely-populated urban areas. Therefore, the system cannot always detect location by wireless LAN base stations. In the future, cellular phone and wireless LAN base stations will be available in almost all urban areas, and be used for detecting the travelers' location.

Table 1: $\quad$ Situations and responses of the system.

\begin{tabular}{|l|l|}
\hline \multicolumn{1}{|c|}{ Actions and situations of users } & \multicolumn{1}{c|}{ Responses of the system } \\
\hline A traveler inputs the schedule. & The system proposes itineraries. \\
\hline A traveler modifies the schedule. & The system proposes revised itineraries. \\
\hline $\begin{array}{l}\text { The system estimates that the user is } \\
\text { going to transfer. } \\
\text { A traveler requests information of the } \\
\text { transfer station. }\end{array}$ & $\begin{array}{l}\text { The system gives information of the map } \\
\text { and timetable. }\end{array}$ \\
\hline $\begin{array}{l}\text { The system estimates that the traveler is } \\
\text { transferring to a bus. } \\
\text { A traveler requests information of a } \\
\text { transfer station. }\end{array}$ & $\begin{array}{l}\text { The system gives the bus timetable the } \\
\text { traveler will get on board the bus. }\end{array}$ \\
\hline $\begin{array}{l}\text { The system estimates that the traveler is } \\
\text { walking. } \\
\text { A traveler requests information of the } \\
\text { surrounding area. }\end{array}$ & The system offers the route map. \\
\hline $\begin{array}{l}\text { The system obtains information of train } \\
\text { operation disturbances. }\end{array}$ & Alert message. \\
\hline
\end{tabular}

Second, the system always needs connections to outside networks in order to get an access to WWW resources to obtain information on train operation disturbances or data from a bus location system, but this is not always the case. For instance, when we are on an underground train, which is running between stations, the system cannot connect to an outside network since in Japan almost all the communication services are not available underground unless you are in one of the stations.

The system consists of a mobile PC, equipped with a GPS unit and a cellular phone communication card. Location data was registered in advance, the system searches route and time by cellular phone network only in case GPS location data is in the registered area. 


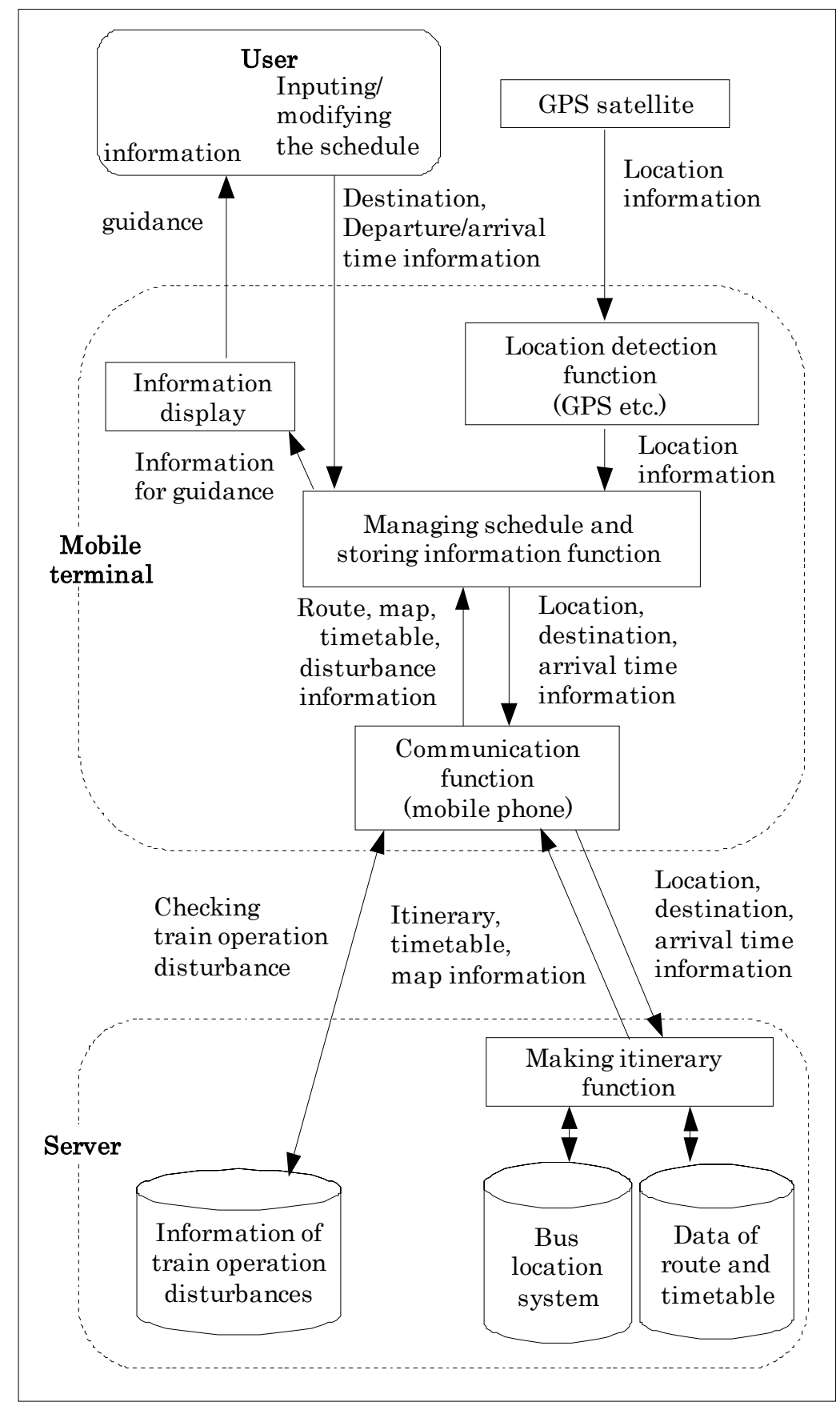

Figure 1: Component of the system. 
The flowchart of the system operation is shown in Fig. 3. After starting guidance, the system checks the status of the GPS signal which it receives. When a GPS signal is available the system compares registered location information with the location information obtained from the GPS, and if the traveler's location is contained in the registered area and the same location of guidance, guidance is continued as it is. If the system detects some sort of inconsistencies between the schedule of the traveler and his/her current location, it revises the travel itinerary accordingly. At last, the guidance ends when the system detects the arrival at the destination.

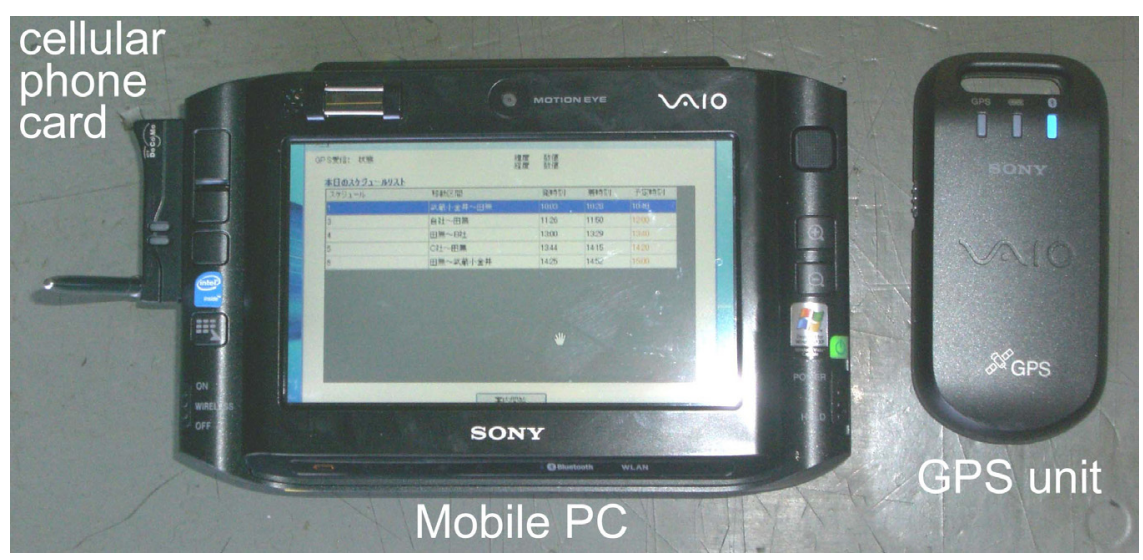

Figure 2: Composition of the prototype system.

\section{Result of experimental operation of the prototype system}

We carried out a field test of the system in one of the Tokyo suburban areas including routes of railways and buses.

The test revealed that the system can provide travelers with appropriate information, which is updated when they enter into one of the pre-registered areas, as long as GPS and cellular phone are available.

On the other hand, in case the accuracy of GPS is insufficient, the system may indicate a location that is different from the actual location. In this case, the system updates guidance undesirably. In some cases when the bus we were getting on encounters a traffic jam and approaches one of the pre-registered areas near a station, the system mistakenly estimated that we arrived at a station and guidance messages related to the transfer to the railway were delivered to us in an inappropriate timing.

Although the result of the experimental operation proved the potential effectiveness of the system, it has also been clarified that we have to improve the accuracy of location detection in order to put the system into real operation. 


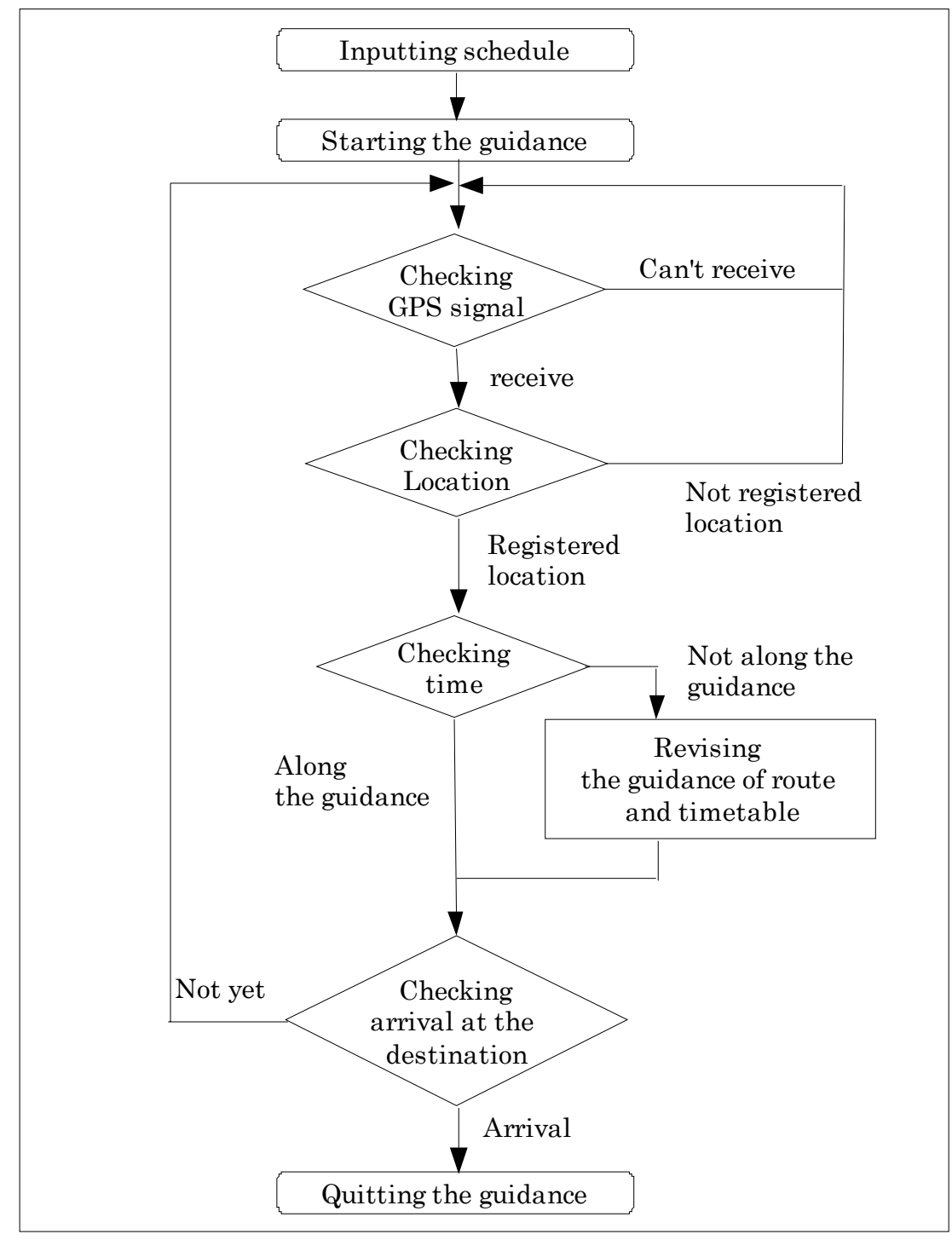

Figure 3: Flowchart of the prototype system.

\section{Conclusion}

In this paper, we presented a schedule-oriented navigation system for travelers. We summarized the concepts and goals of the system as well as its basic architecture.

The system has five major functions. First, the system provides travelers with an appropriate itinerary taking into consideration their activity schedule and their 
current location. Second, the system rearranges itineraries promptly in response to any updates of an activity schedule and the location of travelers. Thirdly, the system automatically creates the user's itinerary for a whole day, taking into consideration his/hers activity schedule. Fourth, the system guides time to spare for a side trip. Fifth, the system utilizes information of the bus location system and railway disturbance for guidance. The bus location system notifies us where a bus is running and when a bus comes to a bus stop. In case the bus location system is available, a transfer between the train and bus will be much smoother. If the system is available for practical use, the convenience of traveler will be greatly improved.

We have developed a first prototype system and carried out a field test in a Tokyo suburban area. The test revealed some remaining issues which are mainly related to the restricted availability and accuracy of location detection technologies. We will study the possibility of combining heterogeneous location technologies into an integrated location system.

We will continue improving and enhancing the system so that it can be operational in the real field of passenger transport in the near future.

\section{References}

[1] B.J. Rhodes, P. Maes, "Just-in Time information retrieval agent", IBM System Journal Vol.39, NOS 3\&4 (2000)

[2] Ogino, T., Tsuchiya, R., Seki, K., Sato, Y., "CyberRail - the fabric of ITS enhanced by railway transport," Proc. $5^{\text {th }}$ World Congress on Railway Research (2001)

[3] Tsuchiya, R., Seki, K., Ogino, T., Sato, Y., "CyberRail: An Enhanced Railway System for Intermodal Transportation", Proc. 8th World Congress on Intelligent Transportation Systems (2001)

[4] Tsuchiya, R., Ogino, T., Matsuoka, A., Goto, Y., "Design and Implementation of Context-aware Passenger Support System", Proc. 11th World Congress on Intelligent Transportation Systems (2004)

[5] Tsuchiya, R., Seki, K., Ogino, T., Matsuoka, A., Goto, K., "CyberRail and its significance in the coming ubiquitous society", Proc. of the $6^{\text {th }}$ World Congress on Railway Research (2003)

[6] Tsuchiya, R., Nozue, M., "Supporting Intermodal Travelers by Agentbased Information Integration". Proc. of the $7^{\text {th }}$ World Congress on Railway Research (2006) 